\title{
Comparison of longissimus dorsi Fatty Acids Profiles in Gansu Black Yak and Chinese Yellow Cattle Steers and Heifers
}

\author{
S. G. Zhang ${ }^{1}$, T. Liu², M. A. Brown ${ }^{2,3}$, and J. P. Wu ${ }^{2 *}$ \\ ${ }^{1}$ Faculty of Food Science and Engineering, Gansu Agricultural University, \\ No. 1 Yingmen Village Anning, Lanzhou, Gansu, PRC 730070 \\ ${ }^{2}$ Faculty of Animal Science and Technology, Gansu Agricultural University, \\ No. 1 Yingmen Village Anning, Lanzhou, Gansu, PRC 730070 \\ ${ }^{3} B \& B$ Research \& Development, LLC, 16835 SW 27th St., El Reno, OK, USA 73036
}

\begin{abstract}
Fatty acid (FA) composition of longissimus dorsi intramuscular fat in Black Yak and Chinese Yellow Cattle were evaluated in 44 Black Yak and 41 Chinese Yellow Cattle of both genders. Interactions of species with gender were observed for total saturated fatty acid (SFA), unsaturated fatty acid (UFA), monounsaturated fatty acid (MUFA), polyunsaturated fatty acid (PUFA), palmitic acid, stearic acid, oleic acid, linolenic acid, arachidonic acid, EPA, and DHA concentrations, as well as PUFA/SFA ratio in the longissimus dorsi ( $p<0.05)$. The SFA percentage was greater in yellow cattle than yak in both genders but the species difference in heifers was greater than in steers $(p<0.05)$. Yak had greater UFA, MUFA and PUFA percentages than yellow cattle in both steers and heifers $(p<0.05)$ but the difference between yak and yellow cattle heifers was greater than yak and yellow cattle steers. The percentages of inolenic acid, arachidonic acid, eicosapentaenoic acid, and docosahexaenoic acid; and PUFA/SFA were greater in yak than yellow cattle in both steers and heifers $(p<0.05)$. In addition, the ratio of $n-6 / n-3$ PUFA in yak was lesser than in yellow cattle $(p<0.05)$. These results indicated that FA composition generally differed between yak and yellow cattle but the differences were not the same in heifers as compared to steers. Results also suggested that species differences in FA composition tended to favor Black Yak over Chinese Yellow Cattle, indicating that the longissimus dorsi of Black Yak may have a higher nutritive value than that of Chinese Yellow Cattle and potential for development as a desirable natural product.
\end{abstract}

Key words: black yak, yellow cattle, gender, longissimus dorsi, fatty acids

Received October 15, 2014; Revised December 12, 2014; Accepted January 14, 2015

\section{Introduction}

Today's consumers are currently searching for safer and healthier foods, with lower fat and cholesterol content and with more favorable fatty acid profiles. Yaks (Poephagus grunniens or Bos grunniens) are regarded as one of the world's most unique domestic food-producing animals. The meat of yak (Bos grunniens) is regarded as one of the world's most contaminant-free natural foods in the world and has high protein (approximately 21\%), low fat and cholesterol, desirable amino acids, and good flavor and other desirable characteristics (Li et al., 2005). These characteristics may result from the unique production environment of yak which include high altitudes with less pollutants, low temperature (annual average $0^{\circ} \mathrm{C}$ ), large

*Corresponding author: J. P. Wu, Gansu Agricultural University, Lanzhou, Gansu 730070, PRC. Tel: +86-931-7631492, E-mail: wujp@gsau.edu.cn temperature differences between day and night (more than $15^{\circ} \mathrm{C}$ ), shorter growing season (110-135 d), strong radiation (more than $140-195 \mathrm{kcal} / \mathrm{cm}^{2}$ per year), and low oxygen partial pressure (less than $110 \mathrm{mmHg}$ ). Hocquette et al. (2010) concluded that pasture-based production systems are characterized by seasonal and geographic variations, due to the abundance and botanical diversity of pasture, and it is expected that meat lipids from these animals would reflect this variability.

The fatty acid profile of meats is of interest, not only because of their important biological functions (e.g., signaling, stabilization, hormone synthesis, formation of cellular membranes, etc.) but also because of their value for human health. The Food and Agriculture Organization of United Nations (FAO), the World Health Organization (WHO) (Burlingame et al., 2009) and, more recently, the European Food Safety Authority (EFSA, 2010) reviewed the specific guidelines for fat and fatty acids for human nutrition. Among the recommendations, a reduction in 
the intake of saturated fatty acids (SFA), trans fatty acids (TFA) and cholesterol, as well as an increase in the intake of n-3 polyunsaturated fatty acids (PUFA), in particular eicosapentaenoic acid (n-3) and docosahexaenoic acid (n3) were recommended (Daley et al., 2010).

In western China, the sources of beef include beef from yak produced at higher altitudes and beef from Bos taurus cattle produced at lower altitudes. Much of the beef from Bos taurus cattle is from indigenous Chinese yellow cattle. Consequently, it is of interest to compare fatty acid profiles of Yak and Chinese Yellow Cattle, each produced in their conventional and obligate production environment. However, gender is also a major factor affecting fatty acid composition of intramuscular depot fats (Eichhorn et al., 1986; Huerta-Leidenz et al., 1993; Yoshimura and Namikawa, 1983; Zembayashi et al., 1995).

Food safety, natural productions systems, and food nutritional value are of increasing interest to Chinese consumers and influence their choices at the marketplace. To provide critical information on the nutritional value of red meat available to Chinese consumers, the objectives of this study were 1) to determine fatty acid profiles in both Black Yak and Chinese Yellow Cattle; 2) to determine gender differences in fatty acid profiles for the Black Yak and Chinese Yellow Cattle; and 3) to determine if gender differences in fatty acid profiles were similar in both species of cattle.

\section{Materials and Methods}

\section{Animal handling and muscle sampling}

In this study forty-four pure Black Yak of both genders (21 steers and 23 heifers, 250-300 kg) from Ma Qu, Gannan, Gansu and forty-one Chinese Yellow Cattle of both genders (20 steers and 21 heifers, 540-600 kg) from Kang Le, Gansu were used, with ages ranging from 2 to 3 years. The yak grazed on cool-season pasture during the grazing season and were fed harvest forages otherwise and the yellow cattle were fed according to local yellow cattle specifications (Table 1). Yak from Ma Qu were managed at higher altitude, and lower mean annual precipitation and mean annual temperature than yellow cattle from Kang Le (Table 2). All animals were harvested in
Table 1. Finishing ration for Chinese Yellow Cattle

\begin{tabular}{ccc}
\hline \hline Item & As-fed percentage & As-fed amount $(\mathrm{kg} / \mathrm{hd} / \mathrm{d})$ \\
\hline Corn & 31 & 1.06 \\
Wheat & 29 & 0.98 \\
Linseed Meal & 10 & 0.35 \\
Wheat bran & 29 & 0.98 \\
Salt & 1 & 0.03 \\
\hline Total & 100 & 3.40 \\
\hline
\end{tabular}

mid-October 2008 in accordance with national standards of humane food animal harvesting and processing. The carcasses chilled for $72 \mathrm{~h}$ before sampling at $4^{\circ} \mathrm{C}$. Samples from one half of the carcass, across the whole width of the muscle, were taken from the longissimus dorsi muscle at the 12-13th rib and individually vacuum-packed, identified by animal number and stored at $-20^{\circ} \mathrm{C}$ until analyzed.

\section{Fatty acid analysis}

For the analysis of fatty acid composition of longissimus dorsi (LD) intramuscular fat was extracted following the procedure of Folch et al. (1957) with some modifications. Briefly, $5 \mathrm{~g}$ of homogenized muscle was blended with chloroform/methanol $(2: 1, \mathrm{v} / \mathrm{v})$ solution, filtered and mixed with saline solution $(0.88 \% \mathrm{NaCl})$ at room temperature. After separation into two phases, the aqueous methanol fraction was discarded and the chloroform lipid fraction was washed with distilled water/methanol (1:1, $\mathrm{v} / \mathrm{v})$. After further filtration and evaporation by means of a rotary evaporator, lipid extracts were transferred to test tubes. Triplicates of $10 \mathrm{mg}$ of lipids were methylated using $2 \mathrm{~mL}$ of hexane and $0.2 \mathrm{~mL}$ of $2 \mathrm{M}$ methanolic $\mathrm{KOH}$ (Liu et al., 2013; Peng et al., 2010). Qualitative and quantitative measurements of fatty acids content were performed by a gas chromatograph (PE Clarus 500, Perkin Elmer, USA) with a flame ion detector (FID). The separation was carried out using a capillary column OV-1701 (50 m $\times$ $0.25 \mathrm{~mm} \times 0.50 \mu \mathrm{m}$ ) with the following temperature program: $55^{\circ} \mathrm{C}$ for $3 \mathrm{~min}$ then $13^{\circ} \mathrm{C} / \mathrm{min}$ to $175^{\circ} \mathrm{C}$ then $4^{\circ} \mathrm{C} /$ $\min$ to $210^{\circ} \mathrm{C}$, held for $20 \mathrm{~min}$; the injector temperature was $270^{\circ} \mathrm{C}$, and the detector temperature was $280^{\circ} \mathrm{C}$. The injection volume was $1 \mu \mathrm{L}$ and the carrier gas was nitrogen. Identification and quantification of the methyl esters

Table 2. Location altitudes, mean annual temperatures, and annual precipitation

\begin{tabular}{cccc}
\hline \hline Location (Breed) & Altitude $(\mathrm{m})$ & Mean Temperature $\left({ }^{\circ} \mathrm{C}\right)$ & Annual Precipitation $(\mathrm{mm})$ \\
\hline Ma Qu (YK) & $3,300-4,806$ & 1.1 & 615.5 \\
Kang Le (YC) & $1,898-3,908$ & 7.0 & 625 \\
\hline
\end{tabular}

${ }^{1}$ YK: Black Yak, YC: Chinese Yellow Cattle. 
of the fatty acids was achieved by comparison with the retention times and concentrations of methyl esters of standard fatty acids. Fatty acids reported in this study include total saturated fatty acid (SFA), unsaturated fatty acid (UFA), monounsaturated fatty acid (MUFA), polyunsaturated fatty acid (PUFA), palmitic acid, stearic acid, oleic acid, $\alpha$-linoleic acid, arachidonic acid, eicosapentaenoic acid (EPA), and docosahexaenoic acid (DHA) concentrations, as well as PUFA/SFA ratio.

\section{Statistical analyses}

The influence of species and gender on fatty acid composition were evaluated using PROC MIXED (SAS, USA). The linear model used was:

$$
\text { Yijk }=\mu+S i+G j+S G i j+e k(i j)
$$

where:

Yijk is the observed value of the $\mathrm{k}_{\mathrm{th}}$ animal in the $\mathrm{i}_{\mathrm{th}}$ species and $j_{\text {th }}$ gender, $\mu$ the mean value common to all observations, Si the fixed effects of the ith species, $\mathrm{Gj}$ the fixed effects of the $j_{\text {th }}$ gender, SGij the fixed interaction between the $i_{\text {th }}$ species and jth gender, and ek (ij) the random deviation of the $k_{t h}$ animal in the ith species and $j_{t h}$ gender. Pairwise comparisons among least squares means were done using $\mathrm{t}$ statistics at $p<0.05$ and $p<0.10$ with $p<0.10$ denoting a trend.

\section{Results and Discussion}

\section{Saturated fatty acids}

In the intramuscular fat of both yak and yellow cattle, the most abundant fatty acids were palmitic acid and stearic acid, both SFA, which together comprised more than $90 \%$ of the total saturated fatty acids. A similar trend was observed by Liu et al. (2013) for longissimus dorsi intramuscular fat from dairy and beef steer and by Zhang et al. (2009) for intramuscular fatty acids of QingHai Datong Yak.

A species $\mathrm{x}$ gender interaction was observed for total SFA percentage in the intramuscular longissimus dorsi fat. The SFA percentage was greater in yellow cattle than yak in both genders $(p<0.05)$ but the species difference in heifers was greater than in steers. The SFA percentage for yellow cattle heifers was greatest $(60.79 \%)$, followed in order by yellow cattle steers $(55.70 \%)$, yak heifers $(52.44 \%)$ and yak steers $(50.23 \%)$. The concentration of SFA was greatest in yellow cattle heifers $(p<0.05)$ compared to other species-gender means. Our results were similar to Zhang et al. (2009) who reported that the SFA concentration in intramuscular fat of Datong yak was lower than that of Datong yellow cattle $(p<0.01)$.

Further, species $\mathrm{x}$ gender interactions were observed for myristic acid, palmitic acid, heptadecanoic acid, stearic acid, and arachidic acid $(p<0.05)$. The myristic acid percentage was greater in yak than yellow cattle in both genders $(p<0.05)$ but the species difference in steers was greater than in heifers. Yellow cattle had greater palmitic acid percentage than yak in both steers and heifers $(p<0.05)$ but the difference between yellow cattle and yak heifers was greater than yellow cattle and yak steers. Similar to myristic acid, heptadecanoic acid percentage was greater in yak than yellow cattle in both steers and heifers $(p<$ 0.05 ) but yak vs. yellow cattle was greater in heifers than steers $(p<0.05)$. Yellow cattle stearic acid percentage exceeded yak in both genders $(p<0.05)$ but the contrast of yellow cattle and yak was greater in steers than in heifers. Comparisons of arachidic acid percentage in both genders favored yak over yellow cattle $(p<0.05)$ but the species difference in heifers was greater than in steers.

With the exceptions of myristic acid and stearic acid, species differences in these saturated fatty acids were larger in heifers than steers. However, while yellow cattle had greater total saturated fatty acids, yak were greater in 3 of these saturated fatty acids (myristic acid, heptadecanoic acid, arachidic acid) and yellow cattle were greater in 2 of these saturated fatty acids (palmitic acid, stearic acid).

Species influenced percentage butyric acid, caproic acid, caprylic acid, capric acid, and tridecanoic acid $(p<0.05)$. The concentrations of butyric acid and capric acid in yak were greater than in yellow cattle $(p<0.05)$. However, the content of caproic acid, caprylic acid and tridecanoic acid in yak were lesser than in yellow cattle $(p<0.05)$. Gender influenced butyric acid, caproic acid, caprylic acid ( $p<$ $0.10)$, and capric acid, lauric acid $(p<0.05)$. The percentage of butyric acid and caprylic acid in heifers were greater than in steers $(p<0.10)$. However, caproic acid, capric acid and lauric acid percentages were lesser in heifers than in steers $(p<0.10, p<0.05)$.

\section{Unsaturated fatty acids}

A species $\mathrm{x}$ gender interaction was observed for total UFA percentage in the intramuscular longissimus dorsi fat. The UFA percentage was greater in yak than yellow cattle in both genders $(p<0.05)$ but the species difference in steers was greater than in steers. The UFA percentage for steers of yak was greatest $(49.42 \%)$, followed in order heifers in yak (46.80\%), heifers $(44.45 \%)$ and steers yel- 
low cattle $(39.05 \%)$. Other results demonstrated that fatty acid composition (percentage of SFA and UFA) of longissimus dorsi muscle was effected by breed, gender and production system in Hereford x Friesian and Angus x Friesian cattle (Enser et al., 1998).

Further, a species $\mathrm{x}$ gender interactions were observed for total MUFA percentage and oleic acid in the intramuscular longissimus dorsi fat. Yak had greater MUFA percentage than yellow cattle in both genders $(p<0.05)$ but the difference between yak and yellow cattle steers was greater than yak and yellow cattle heifers. Enser et al. (1998) reported that oleic acid can reduce low-density lipoprotein cholesterol to prevent arteriosclerosis without decreasing the level of high-density lipoprotein cholesterol beneficial to the human body. In our study, steers of both species provided similar levels of oleic acid but yak heifers had greater percentage oleic acid compared to yellow cattle heifers $(p<0.05)$.

Breed influenced palmitoleic acid content in longissimus dorsi muscle $(p<0.05)$. The concentration of palmi- toleic acid in yak was greater than in yellow cattle $(p<$ 0.05). The result was similar with Zhang et al. (2009) who reported the palmitoleic acid content of Datong yak was higher than that of yellow cattle $(p<0.01)$.

Gender also influenced the concentration of palmitoleic acid in our study. Heifer palmitoleic acid content was greater than steers $(p<0.05)$. Gender differences in the fatty acid composition are known to be associated with hormonal changes and their influence on enzymatic systems (Prior et al., 1983).

A species $\mathrm{x}$ gender interaction was observed for total PUFA percentage in the intramuscular longissimus dorsi fat. Yak PUFA percentage exceeded yellow cattle in steers and heifers $(p<0.05)$ but the contrast of yellow cattle and yak was greater in steers than heifers. The concentration of linolenic acid, arachidonic acid, eicosapentaenoic acid, and docosahexaenoic acid were also demonstrated species $\mathrm{x}$ gender interactions $(p<0.05)$. For linolenic acid, the percentage was greater in yak than yellow cattle in both genders $(p<0.05)$ but the species difference in heifers was

Table 3. Least squares means and standard errors for fatty acid composition of longissimus dorsi from Yak and Yellow cattle

\begin{tabular}{|c|c|c|c|c|c|c|}
\hline \multirow{2}{*}{\multicolumn{2}{|c|}{ FAME }} & \multicolumn{2}{|c|}{ Yak } & \multicolumn{2}{|c|}{ Yellow cattle } & \multirow{2}{*}{ SEM } \\
\hline & & Female & Male & Female & Male & \\
\hline $\mathrm{C} 4: 0$ & Butyric Acid & 0.35 & 0.32 & 0.29 & 0.25 & 0.02 \\
\hline C6:0 & Caproic Acid & 0.33 & 0.37 & 0.4 & 0.44 & 0.02 \\
\hline $\mathrm{C} 8: 0$ & Caprylic Acid & 0.39 & 0.35 & 0.54 & 0.49 & 0.02 \\
\hline C10:0 & Capric Acid & 0.48 & 0.54 & 0.43 & 0.49 & 0.02 \\
\hline C12:0 & Lauric Acid & 0.14 & 0.17 & 0.11 & 0.15 & 0.01 \\
\hline C13:0 & Tridecanoic Acid & 0.02 & 0.03 & 0.37 & 0.4 & 0.01 \\
\hline C14:0 & Myristic Acid & $0.76^{\mathrm{a}}$ & $0.81^{\mathrm{a}}$ & $0.57^{\mathrm{b}}$ & $0.45^{\mathrm{c}}$ & 0.04 \\
\hline C16:0 & Palmitic acid & $22.65^{b}$ & $18.87^{\mathrm{c}}$ & $30.96^{\mathrm{a}}$ & $21.86^{b}$ & 0.4 \\
\hline C17:0 & Heptadecanoic Acid & $0.36^{\mathrm{a}}$ & $0.31^{\mathrm{b}}$ & $0.04^{\mathrm{c}}$ & $0.05^{\mathrm{c}}$ & 0.01 \\
\hline C18:0 & Stearic acid & $25.43^{c}$ & $26.93^{b}$ & $26.96^{\mathrm{b}}$ & $30.97^{\mathrm{a}}$ & 0.4 \\
\hline C19:0 & Nonadecanoic acid & 1.2 & 1.24 & 0.01 & 0.02 & 0.05 \\
\hline C20:0 & Eicosanoic acid & $0.33^{\mathrm{a}}$ & $0.30^{\mathrm{a}}$ & $0.10^{\mathrm{b}}$ & $0.14^{\mathrm{b}}$ & 0.02 \\
\hline SFA & saturated fatty acid & $52.44^{\mathrm{c}}$ & $50.23^{d}$ & $60.79^{a}$ & $55.70^{\mathrm{b}}$ & 0.62 \\
\hline C16:1 & Palmitoleic Acid & $5.75^{\mathrm{a}}$ & $4.41^{\mathrm{b}}$ & $3.97^{\mathrm{a}}$ & $2.63^{\mathrm{b}}$ & 0.17 \\
\hline C18:1 (n-9) & Oleic Acid & $33.43^{\mathrm{b}}$ & $35.97^{\mathrm{a}}$ & $28.77^{\mathrm{c}}$ & $36.03^{\mathrm{a}}$ & 0.31 \\
\hline MUFA & monounsaturated fatty acid & $39.18^{\mathrm{b}}$ & $40.38^{\mathrm{a}}$ & $32.74^{\mathrm{c}}$ & $38.66^{\mathrm{b}}$ & 0.38 \\
\hline C18:2 (n-6) & Linoleic Acid & 5.69 & 5.34 & 4.59 & 4.22 & 0.17 \\
\hline C18:3 (n-6) & Linolenic Acid & $0.16^{\mathrm{a}}$ & $0.09^{\mathrm{b}}$ & $0.05^{\mathrm{c}}$ & $0.01^{\mathrm{d}}$ & 0.01 \\
\hline $\mathrm{C} 20: 4(\mathrm{n}-6)$ & Lignoceric Acid & $1.15^{\mathrm{b}}$ & $2.97^{\mathrm{a}}$ & $1.27^{\mathrm{b}}$ & $1.21^{\mathrm{b}}$ & 0.1 \\
\hline $\mathrm{C} 20: 5(\mathrm{n}-3)$ & cis-5,8,11,14,17-Eicosapentaenoic Acid & $0.22^{\mathrm{b}}$ & $0.30^{\mathrm{a}}$ & $0.06^{\mathrm{c}}$ & $0.04^{\mathrm{c}}$ & 0.01 \\
\hline $\mathrm{C} 22: 6(\mathrm{n}-3)$ & cis-4,7,10,13,19-Docosahexaenoic Acid & $0.49^{\mathrm{a}}$ & $0.40^{\mathrm{b}}$ & $0.21^{\mathrm{c}}$ & $0.24^{\mathrm{c}}$ & 0.02 \\
\hline PUFA & polyunsaturated fatty acid & $7.61^{\mathrm{b}}$ & $9.03^{\mathrm{a}}$ & $5.30^{\mathrm{c}}$ & $5.79^{\mathrm{d}}$ & 0.21 \\
\hline UFA & unsaturated fatty acid & $46.80^{\mathrm{b}}$ & $49.42^{\mathrm{a}}$ & $40.05^{\mathrm{d}}$ & $44.45^{\mathrm{c}}$ & 0.46 \\
\hline MUFA/SFA & monounsaturated fatty acid/saturated fatty acid & $0.75^{\mathrm{b}}$ & $0.81^{\mathrm{a}}$ & $0.54^{\mathrm{d}}$ & $0.07^{\mathrm{c}}$ & 0.01 \\
\hline PUFA/SFA & polyunsaturated fatty acid/saturated fatty acid & $0.15^{\mathrm{b}}$ & $0.18^{\mathrm{a}}$ & $0.09^{\mathrm{c}}$ & $0.10^{\mathrm{c}}$ & 0.01 \\
\hline n-6 PUFA & n-6 polyunsaturated fatty acid & $6.84^{\mathrm{b}}$ & $8.31^{\mathrm{a}}$ & $5.86^{\mathrm{c}}$ & $5.42^{c}$ & 0.2 \\
\hline n-3 PUFA & n-3 polyunsaturated fatty acid & 0.77 & 0.72 & 0.44 & 0.37 & 0.03 \\
\hline$n-6 / n-3 P U F A$ & n-6/n-3 polyunsaturated fatty acid & 9.38 & 12.36 & 13.38 & 15.32 & 0.67 \\
\hline
\end{tabular}

${ }^{\mathrm{a}-\mathrm{d}}$ Species $\mathrm{x}$ gender means on the same row with differing superscripts differ $(p<0.05)$. 
Table 4. Least squares means and standard errors for fatty acid composition of longissimus dorsi from Yak and Yellow cattle

\begin{tabular}{|c|c|c|c|c|c|c|}
\hline \multirow{2}{*}{\multicolumn{2}{|c|}{ FAME }} & \multicolumn{4}{|c|}{ Main Effect Means } & \multirow{3}{*}{$\begin{array}{c}\text { SEM } \\
0.01\end{array}$} \\
\hline & & Yak & Yellow cattle & Female & Male & \\
\hline $\mathrm{C} 4: 0$ & Butyric Acid & $0.34^{\mathrm{w}}$ & $0.27^{x}$ & $0.32^{\mathrm{Y}}$ & $0.29^{Z}$ & \\
\hline C6:0 & Caproic Acid & $0.35^{\mathrm{x}}$ & $0.42^{\mathrm{w}}$ & $0.37^{\mathrm{Z}}$ & $0.40^{\mathrm{Y}}$ & 0.01 \\
\hline C8:0 & Caprylic Acid & $0.37^{\mathrm{x}}$ & $0.51^{\mathrm{w}}$ & $0.46^{\mathrm{Y}}$ & $0.42^{Z}$ & 0.02 \\
\hline C10:0 & Capric Acid & $0.51^{\mathrm{w}}$ & $0.46^{\mathrm{x}}$ & $0.46^{\mathrm{z}}$ & $0.51^{\mathrm{y}}$ & 0.02 \\
\hline C12:0 & Lauric Acid & 0.15 & 0.13 & $0.13^{\mathrm{z}}$ & $0.16^{\mathrm{y}}$ & 0.01 \\
\hline $\mathrm{C} 13: 0$ & Tridecanoic Acid & $0.02^{\mathrm{x}}$ & $0.38^{\mathrm{w}}$ & 0.19 & 0.21 & 0.01 \\
\hline C14:0 & Myristic Acid & 0.78 & 0.51 & 0.66 & 0.63 & 0.03 \\
\hline C16:0 & Palmitic acid & 20.76 & 26.41 & 26.81 & 20.36 & 0.28 \\
\hline C17:0 & Heptadecanoic Acid & 0.33 & 0.04 & 0.2 & 0.18 & 0.01 \\
\hline C18:0 & Stearic acid & 26.18 & 28.97 & 26.2 & 28.95 & 0.28 \\
\hline C19:0 & Nonadecanoic acid & 1.22 & 0.02 & 0.61 & 0.63 & 0.04 \\
\hline C20:0 & Eicosanoic acid & 0.32 & 0.12 & 0.22 & 0.22 & 0.01 \\
\hline SFA & saturated fatty acid & 51.34 & 58.24 & 56.62 & 52.97 & 0.43 \\
\hline C16:1 & Palmitoleic Acid & $5.08^{w}$ & $3.30^{\mathrm{x}}$ & $4.86^{\mathrm{y}}$ & $3.52^{\mathrm{z}}$ & 0.12 \\
\hline C18:1 (n-9) & Oleic Acid & 34.7 & 32.4 & 31.1 & 36 & 0.22 \\
\hline MUFA & monounsaturated fatty acid & 39.78 & 35.7 & 35.96 & 39.52 & 0.27 \\
\hline $\mathrm{C} 18: 2(\mathrm{n}-6)$ & Linoleic Acid & $5.52^{\mathrm{w}}$ & $4.40^{\mathrm{x}}$ & $5.14^{\mathrm{y}}$ & $4.78^{\mathrm{z}}$ & 0.12 \\
\hline $\mathrm{C} 18: 3(\mathrm{n}-6)$ & Linolenic Acid & 0.13 & 0.03 & 0.05 & 0.11 & 0.01 \\
\hline $\mathrm{C} 20: 4(\mathrm{n}-6)$ & Lignoceric Acid & 2.06 & 1.24 & 1.21 & 2.09 & 0.07 \\
\hline $\mathrm{C} 20: 5(\mathrm{n}-3)$ & cis-5,8,11,14,17-Eicosapentaenoic Acid & 0.26 & 0.05 & 0.14 & 0.17 & 0.01 \\
\hline$C 22: 6(n-3)$ & cis-4,7,10,13,19-Docosahexaenoic Acid & 0.45 & 0.22 & 0.35 & 0.32 & 0.01 \\
\hline PUFA & polyunsaturated fatty acid & 8.32 & 6.05 & 6.96 & 7.41 & 0.14 \\
\hline UFA & unsaturated fatty acid & 48.11 & 41.75 & 42.92 & 46.93 & 0.32 \\
\hline MUFA/SFA & monounsaturated fatty acid/saturated fatty acid & 0.78 & 0.62 & 0.64 & 0.75 & 0.01 \\
\hline PUFA/SFA & polyunsaturated fatty acid/saturated fatty acid & 0.16 & 0.1 & 0.12 & 0.14 & 0.01 \\
\hline n-6 PUFA & $\mathrm{n}-6$ polyunsaturated fatty acid & 7.58 & 5.64 & 6.35 & 6.87 & 0.15 \\
\hline n-3 PUFA & n-3 polyunsaturated fatty acid & $0.74^{\mathrm{w}}$ & $0.40^{\mathrm{x}}$ & $0.61^{\mathrm{y}}$ & $0.54^{\mathrm{z}}$ & 0.02 \\
\hline n-6/n-3 PUFA & $n-6 / n-3$ polyunsaturated fatty acid & $10.87^{x}$ & $14.35^{\mathrm{w}}$ & $11.38^{\mathrm{z}}$ & $13.84^{y}$ & 0.48 \\
\hline
\end{tabular}

${ }_{\mathrm{w}, \mathrm{x}}$ Species main effect means on the same line with differing superscripts differ $(p<0.05)$.

${ }^{y, z}$ Gender main effect means on the same line with differing superscripts $\operatorname{differ}(p<0.05)$.

${ }^{\mathrm{Y}, \mathrm{Z}}$ Gender main effect means on the same line with differering superscripts differ $(p<0.10)$.

greater than in steers. The concentration of arachidonic acid was similar for yak and yellow cattle heifers but yak steers had greater percentage arachidonic acid than yellow cattle steers $(p<0.05)$. The percentage of EPA was the greatest in both steers and heifers $(p<0.05)$ and it was greater in steers than in heifers from yak $(p<0.05)$. Yak exceed yellow cattle in DHA content in both steers and heifers but the species difference was greatest in heifers $(p<0.05)$. Specie influenced linoleic acid content and it was greater in yak than in yellow cattle $(p<0.05)$. In addition, gender also influenced the concentration of linoleic acid in our study, with heifer linoleic acid percentage greater than steers $(p<0.05)$. Similar to the results of this study, Liu et al. (2010) reported that percentage linoleic acid, linolenic acid, arachidonic acid, EPA, and DHA were greater in yak than in yellow cattle from mesentery and kidney fat $(p<0.05, p<0.01)$. The results for EPA and DHA may be explained from the fact that essential fatty acid of linoleic acid is derived entirely from the diet and the animal possesses desaturase and elongase enzymes that can convert linoleic acid to longer-chain n-6 fatty acids such as arachidonic acid. Similarly, the linolenic acid is present in the leaves of plants and grasses and it can be converted to long-chain n-3 fatty acid such as EPA and DHA (Williams and Burdge, 2006).

Further, Species x gender interactions were observed for the ratio of MUFA/SFA and PUFA/SFA in the intramuscular longissimus dorsi fat. The ratio of MUFA/SFA was greater in yak than in yellow cattle in both genders $(p<0.05)$ but the species difference in steers was greater than in heifers. The U. K. Department of Health (1994) recommended that ratios between these fatty acid groups should be $>0.4$ for PUFA/SFA. In our study, the ratios of PUFA/SFA were all $<0.4$ both in yak and yellow cattle. However, the ratio of PUFA/SFA was greater in yak than yellow cattle in both steers and heifers $(p<0.05)$. There 
was also a species $x$ gender interaction in percent $n-6$ PUFA $(p<0.05)$. Yak had greater n-6 PUFA percentage than yellow cattle in both steers and heifers $(p<0.05)$ but the difference between yellow cattle and yak steers was greater than yellow cattle and yak steers. This result may reflect differences noted in the concentration of linoleic acid, which is converted to n-6 by desaturase and elongase enzymes (Williams and Burdge, 2006).

Breed influenced n-3 PUFA percentage and the ratio of n-6/n-3 PUFA in the intramuscular longissimus dorsi muscle fat $(p<0.05)$. Yak had greater the concentration of $\mathrm{n}$ 3 PUFA than in yellow cattle $(p<0.05)$ and the ratio of $n-$ 6/n-3 PUFA in yak was lesser than in yellow cattle $p<$ $0.05)$. These results were similar to those of Liu et al. (2010). Differences in $n-3$ and n-6/n-3 could be attributable to genetic background of the animals, similar to results reported by Cifuni et al. (2004) or to differences in the diet. Enser et al. (1998) compared the lipid composition of beef intramuscular fat from pasture and concentrate fed animals and concluded that the $n-6 / n-3$ ratio in pasture-fed animals was lesser than in concentrated-fed.

Gender also influenced the concentration of n-3 PUFA and the ratio of $n-6 / n-3$ PUFA in our study. There was a trend for percentage n-3 PUFA in heifers to exceed that of steers $(p<0.10)$. Similarly, there was a trend for the ratio of n-6/n-3 PUFA in heifers to be lesser to that of steers $(p<0.10)$. These results suggest that the $n-3$ and $n-6 / n-3$ ratios in the longissimus dorsi of heifers are more desirable than that of steers, considering human health.

\section{Conclusions}

This study clearly indicates that the fatty acid composition of intramuscular fat from the longissimus dorsi in yak and yellow cattle is significantly affected by species and gender. Differences in finishing diet likely contributed to differences in fatty acid profiles between yak and yellow cattle. The results suggest that the nutritive value of fatty acid profiles between yak and yellow cattle generally favored yak over local yellow cattle but the advantage of yak was more pronounced in one or the other gender, depending on the specific fatty acid. The ratios of PUFA/SFA and n-6/n-3PUFA of yak in the intramuscular fat of the longissimus dorsi muscle in both genders were closer to recommended values than in yellow cattle. However, PUFA/SFA favored yak steers while n-6/n-3 favored yak heifers. Consequently, development of specific products with desirable fatty acid profiles must consider both species and gender. Further research is needed to evaluate species and gender differences for other meat quality attributes, such as tenderness and flavor.

\section{Acknowledgements}

This study was funded by two projects (GNSW-201004, GNSW2011-27).

\section{References}

1. Burlingame, B., Nishida, C., Uauy, R., and Weisell, R. (2009) Fats and fatty acids in human nutrition (Report of a Joint FAO/ WHO Expert Consultation, November 2008). Ann. Nutr. Metab. 55, 1-308.

2. Daley, C. A., Abbott, A., Doyle. P. S., Nader, G. A., and Larson, S. (2010) A review of fatty acids profiles and antioxidant content in grass-fed and grain-fed beef. Nutr. J. 9, 1-12.

3. Department of Health. (1994) Report on health and social subjects, No.46. Nutritional aspects of cardiovascular disease, Her Majesty's Stationery Office, London.

4. EFSA (2010) European Food Safety Authority (EFSA) Scientific opinion on dietary reference values for fats, including saturated fatty acids, polyunsaturated fatty acids, monounsaturated fatty acids, trans fatty acids, and cholesterol. EFSA J. 8, 1-1461.

5. Eichhorn, J. M., Coleman, L. J., Wakayama, E. J., Blomquist, G. J., Bailey, C. M., and Jenkins, T. G. (1986) Effects of breed type and restricted versus ad libitum breeding on fatty acid composition and cholesterol content of muscle and adipose tissue from mature bovine females. J. Anim. Sci. 63, 781-794.

6. Enser, M., Hallet, K. G., Heweet, B., Fursey, G. A. J., Wood, J. D., and Harrington, G. (1998) Fatty acid content and composition of UK beef and lamb muscle in relation to production system and implications for human nutrition. Meat Sci. 49, 329-341.

7. Hocquette, J. F., Gondret, F., Baéza, E., Meédale, F., Jurie, C., and Pethick, D. W. (2010) Intramuscular fat content in meat-producing animals: Development, genetic and nutritional control, and ideninfication of putative markers. Animal 4, 303-319.

8. Huerta-Leidenz, N. O., Cross, H. R., Savell, J. W., Lunt, D. K., Baker, J. F., Pelton, L. S., and Smith, S. B. (1993) Comparison of the fatty acid composition of subcutaneous adipose tissue from mature Brahman and Hereford cows. $J$. Anim. Sci. 71, 625-630.

9. Liu, T., Lei, Z. M., Wu, J. P., and Brown, M. A. (2013) Fatty acid composition differences between adipose depot sites in dairy and beef steer breeds. J. Food Sci. Technol. 1-7.

10. Liu, T., Wu, J. P., Zhang, S. G., and Zhang, Y. B. (2010) Analysis of mesentery fat and perinephrit fat fatty acid composition of yak in Maqu and yellow cattle in Kangle. Sci. Technol. Food Ind. 31, 111-114.

11. Peng, Y. S., Brown, M. A., Wu, J. P., and Liu, Z. (2010) Different oilseed supplements alter fatty acid composition of different adipose tissues of adult ewes. Meat Sci. 85, 542- 
549.

12. Prior, R. L., Smith, S. B., Schanbacher, R. D., and Mersmann, H. J. (1983) Lipid metabolism in finishing bulls and steers implanted with oestradiol-17 $\beta-\alpha$ : propionate. Anim. Prod. 37, 81-85.

13. Webb, E. C., Smet, S. D., Nevel, C. V., Martens, B., and Demeyer, D. I. (1998) Effect of anatomical location on the composition of fatty acids in double-muscled Belgian blue cows. Meat Sci. 50, 45-53.

14. Williams, C. M. and Burdge, G. (2006) Long-chain n-3 PUFA: plant v. marine sources. Proc. Nutr. Soc. 65, 42-50.

15. Yoshimura, T. and Namikawa, K. (1983) Influence of breed, sex and anatomical location on lipid and fatty acid composi- tion of bovine subcutaneous fat. Jpn. J. Zootech. Sci. 54, 97101.

16. Yu, Q., Jiang, Y., Wang, C., and Li, P. (2005) Analysis and Evaluation of the components and flavoring substances in white yak's meat. J. Chinese Inst. Food Sci. Technol. 5, 124127.

17. Zembayashi, M., Nishimura, K., Lunt, D. K., and Smith, S. B. (1995) Effect of breed type and sex on the fatty acid composition of subcutaneous and intramuscular lipids of finishing steers and heifers. J. Anim. Sci. 73, 3325-3332.

18. Zhang, Y. H., Yan, P., Liang, C. N., and Zeng, Y. F. (2009) Composition analysis on intermuscular fatty acid of Qinghai Datong Yak. Agric. Sci. Technol. 10, 145-148. 